\title{
Similarity Form, Similarity Variational Element and Similarity Solution to $(2+1)$ Dimensional Breaking Solition Equation
}

\author{
Lichun $\mathrm{Pu}^{1}$ \\ ${ }^{1}$ College of Optoelectronic Information, Chongqing University of Technology, Chongqing, China \\ Correspondence: Lichun Pu, College of Optoelectronic Information, Chongqing University of Technology, 24 \\ Hongguang Road, Banan Area, Chongqing 400054, China. Tel: 86-23-6256-3271. E-mail:cqplc@cqut.edu.cn
}

Received: May 23, 2012 Accepted: June 10, 2012 Online Published: July 20, 2012

doi:10.5539/jmr.v4n4p81 URL: http://dx.doi.org/10.5539/jmr.v4n4p81

\begin{abstract}
In this paper, the thoughts and methods of ordinary deferential equation of dimensional non-linear partial deferential equation with $(2+1)$ dimensional non-linear partial deferential equation will be analysed first, then the authors deduce the universal similarity form and similarity variational element of $(2+1)$ dimensional breaking solution equation and its suitable deferential equation and find the similarity solition to $(2+1)$ dimensional breaking solition equation.
\end{abstract}

Keywords: $(2+1)$ dimensional breaking solition equation, similarity form, similarity variational element, similarity solition

\section{Introduction}

R. S. Ward once had a conjecture; All of the integrable equations could be got from their dual Y-M equation (or their Promotion Equation) through reduction. It is obviously that document (Li, 1999) had reduced a $(2+1)-$ dimensional breaking soliton equation by using the consistency condition of the dual Y-M equation themselves, through analysis on Variable Soliton Equation. Considering about the document (Guo, 1998), there is an analysis about $1+1$ D nonlinear partial differential equations' similar solution and Paul Painlev's Factor of its correspondence ordinary differential equation, such as Boussinesq Equation, Burgers Equation, KDV equation and Linear Schrodinger Equation. It is a fact that points out when ordinary differential equation own Paul Painlev's Factor, the equation could be integrated. Scientists have focused on researching Soliton solution, Elliptic function solution, Hyperbolic function solution, Sure solution and exact solution of Nonlinear Partial Differential Equations by the way of Line wave method, inverse scattering method, Backlund alternate method and The homogeneous balance method. Furthermore the document $(\mathrm{Gu}, 1990)$ makes a contribution to solving the $(2+1)$-dimensional nonlinear partial differential equations according to Hirota alternate method and Darboux alternate method.In addition, the document (Zhang \& Guo, 2003) has got much Soliton solution from the $(2+1)$-dimensional nonlinear partial differential equations by expanding the Hirota bi-linearity method; by the other hand, it is a fact that the document (Cao et al., 2012; Lei, Ma, \& Fang, 2011; Zhang \& Guo, 2003; Zhang, Huang \& Zheng, 2002; Ruan \& Chen, 2003) have analysis on Soliton solution and Solitary wave structure of $(2+1)$-dimensional nonlinear partial differential equations from varies view with different methods. Having got analytic solutions and similar reduced equation of $(2+1)$-dimensional Camassa-Holm Equation under the condition of ordinary differential equation by Zheng Chunlong. While solving a partial differential equation with method of Scattering inversion, then the differential equation which is corresponding to the partial differential equation would have owned Paul Painlev's Factor; so that research on the Cloning transform, Soliton solution and Equation integrality of the partial differential equation can be done, by M. J. Ablowitz's consideration, the famous professor of Soliton problem. At present, this paper had a research on solving $(2+1)$ nonlinear partial differential equations by Paul Painlev's Factor of differential equations, while no one has ever used to do it. Furthermore, it reduced the ordinary similar form and similar argument of $(2+1)$-dimensional breaking soliton equation by three rules according to the paper, consequently solving the equation's 'similar solution' and Paul Painlev's Factor of its corresponding differential equation; and it has proved the viewpoint by M. J. Ablowitz indirectly. 


\section{The Direct Method and the Direct Transformation of $(2+1)$ Dimensional Breaking Solition}

Equations $(2+1)$ the D breaking soliton Equation 1:

$$
U_{x t}=4 U_{x y} \cdot U_{x}+2 U_{x x} U_{y}-U_{x x x y}
$$

The Equation 1 can be solved by the theory of Similarity methods:

$$
\left\{\begin{array}{l}
U(x, y, t)=\eta(x, y, t)+\zeta(x, y, t) \Omega(z) \\
z=z(x, y, t)
\end{array}\right.
$$

In Equation 2, $\eta(x, y, t), \zeta(x, y, t)$ is the undetermined function, and $\Omega(z)$ is the function of $z$. From Equation 2, it will be:

$$
\begin{aligned}
& \left\{\begin{array}{l}
U_{x}=\eta_{x}+\zeta \cdot \Omega_{z} \cdot z_{x}+\zeta_{x} \cdot \Omega \\
U_{y}=\eta_{y}+\zeta \cdot \Omega_{z} \cdot z_{y}+\zeta_{y} \cdot \Omega \\
U_{x x}=\eta_{x x}+\zeta \cdot \Omega z z \cdot z_{x}^{2}+\left(2 \zeta_{x} \cdot z_{x}+\zeta \cdot z_{x x}\right) \Omega_{z}+\zeta_{x x} \cdot \Omega \\
U_{x y}=\eta_{x y}+\zeta \cdot z_{x} \cdot z_{y} \cdot \Omega_{z z}+\left(\zeta_{x} \cdot z_{y}+\zeta_{y} \cdot z_{x}+\zeta \cdot z_{x y}\right) \Omega_{z}+\zeta_{x y} \cdot \Omega \\
U_{x t}=\eta_{x t}+\zeta \cdot z_{x} \cdot z_{t} \cdot \Omega z z+\left(\zeta_{x} \cdot z_{t}+\zeta_{t} \cdot z_{x}+\zeta \cdot z_{x t}\right) \Omega_{z}+\zeta_{x t} \cdot \Omega
\end{array}\right. \\
& U_{x y} \cdot U_{x}=\zeta^{2} \cdot z_{x}^{2} \cdot z_{y} \cdot \Omega_{z} \cdot \Omega_{z z}+\zeta \cdot \zeta_{x} \cdot z_{x} \cdot z_{y} \cdot \Omega \cdot \Omega_{z z}+\eta_{x} \cdot \zeta \cdot z_{x} \cdot z_{y} \cdot \Omega z z+\zeta_{x} \cdot \zeta_{x y} \Omega^{2} \\
& +\left(\zeta \cdot \zeta_{x} \cdot z_{x} \cdot z_{y}+\zeta \cdot \zeta_{y} \cdot z_{x}^{2}+\zeta^{2} \cdot z_{x} \cdot z_{x y}\right) \Omega_{z}^{2}+\left(\zeta \cdot \zeta_{x y} \cdot z_{x}+\zeta_{x}^{2} \cdot z_{y}+\zeta_{x} \cdot \zeta_{y} \cdot z_{x}+\zeta \cdot \zeta_{x} \cdot z_{x y}\right) \Omega \cdot \Omega_{z} \\
& +\left(\eta_{x} \cdot \zeta_{x} \cdot z_{y}+\eta_{x} \cdot \zeta_{y} \cdot z_{x}+\eta_{x} \cdot \zeta \cdot z_{x y}+\eta_{x y} \cdot \zeta \cdot z_{x}\right) \Omega_{z}+\left(\eta_{x} \cdot \zeta_{x y}+\eta_{x y} \cdot \zeta_{x}\right) \Omega+\eta_{x} \cdot \eta_{x y} \\
& U_{y} \cdot U_{x x}=\zeta^{2} \cdot z_{x}^{2} \cdot z_{y} \cdot \Omega_{z} \cdot \Omega_{z z}+\zeta \cdot \zeta_{y} \cdot z_{x}^{2} \cdot \Omega \cdot \Omega_{z z}+\eta_{y} \cdot \zeta \cdot z_{x}^{2} \cdot \Omega_{z z}+\zeta_{y} \cdot \zeta_{z z} \Omega^{2} \\
& +\left(2 \zeta \cdot \zeta_{x} \cdot z_{x} \cdot z_{y}+\zeta^{2} \cdot z_{y} \cdot z_{x x}\right) \Omega_{z}^{2}+\left(2 \zeta_{x} \cdot \zeta_{y} \cdot z_{x}+\zeta \cdot \zeta_{y} \cdot z_{x x}+\zeta \cdot \zeta_{x x} \cdot z_{y}\right) \Omega \cdot \Omega_{z} \\
& +\left(2 \eta_{y} \cdot \zeta \cdot z_{x}+\eta_{y} \cdot \zeta \cdot z_{x x}+\eta_{x x} \cdot \zeta \cdot z_{y}\right) \Omega_{z}+\left(\eta_{y} \cdot \zeta_{x x}+\eta_{x x} \cdot \zeta_{y}\right) \Omega+\eta_{y} \cdot \eta_{x x} \\
& U_{x x x y}=\zeta \cdot z_{x}^{3} \cdot z_{y} \cdot \Omega_{z z z z}+\left(3 \zeta_{x} \cdot z_{x}^{2} \cdot z_{y}+\zeta_{y} \cdot z_{x}^{3}+3 \zeta \cdot z_{x}^{2} \cdot z_{x y}+3 \zeta \cdot z_{x} \cdot z_{y} \cdot z_{x x}\right) \Omega_{z z z} \\
& +\left(3 \zeta_{x x} \cdot z_{x} \cdot z_{y}+3 \zeta_{x y} \cdot z_{x}^{2}+6 \zeta \cdot z_{x} \cdot z_{x y}+3 \zeta_{x} \cdot z_{y} \cdot z_{x x}+3 \zeta_{y} \cdot z_{x} \cdot z_{x x}+3 \zeta \cdot z_{x y} \cdot z_{x x}\right. \\
& \left.+3 \zeta \cdot z_{x} \cdot z_{x x y}+\zeta \cdot z_{y} \cdot z_{x x x}\right) \Omega_{z z}+\left(\zeta_{x x x} \cdot z_{x}+3 \zeta_{x x y} \cdot z_{x}+3 \zeta_{x x} \cdot z_{x y}+3 \zeta_{x y} \cdot z_{x x}\right. \\
& \left.+3 \zeta_{x} \cdot z_{x x y}+\zeta_{y} \cdot z_{x x x}+\zeta \cdot z_{x x x y}\right) \Omega_{z}+\zeta_{x x x y} \cdot \Omega+\eta_{x x x y}
\end{aligned}
$$

Type the Equations 3, 4, 5, 6 into Equation 1 and settled:

$$
\begin{aligned}
& \zeta \cdot z_{x}^{3} \cdot z_{y} \cdot \Omega_{z z z z}+\left(3 \zeta_{x} \cdot z_{x}^{2} \cdot z_{y}+\zeta_{y} \cdot z_{x}^{3}+3 \zeta \cdot z_{x}^{2} \cdot z_{x y}+3 \zeta \cdot z_{x} \cdot z_{y} \cdot z_{x x}\right) \Omega_{z z z} \\
& -6 \zeta^{2} \cdot z_{x}^{2} \cdot z_{y} \cdot \Omega_{z} \cdot \Omega_{z z}-2\left(2 \zeta \cdot \zeta_{x} \cdot z_{x} \cdot z_{y}+\zeta \cdot \zeta_{y} \cdot z_{x}^{2}\right) \Omega \cdot \Omega_{z z}+\left(3 \zeta_{x x} \cdot z_{x} \cdot z_{y}\right. \\
& +3 \zeta_{x y} \cdot z_{x}^{2}+6 \zeta_{x} \cdot z_{x} \cdot z_{x y}+3 \zeta_{x} \cdot z_{y} \cdot z_{x x}+3 \zeta_{y} \cdot z_{x} \cdot z_{x x}+3 \zeta \cdot z_{x y} \cdot z_{x x}+3 \zeta \cdot z_{x} \cdot z_{x x y} \\
& \left.+\zeta \cdot z_{y} \cdot z_{x x x}+\zeta \cdot z_{x} \cdot z_{t}-4 \eta_{x} \cdot \zeta \cdot z_{x} \cdot z_{y}-2 \eta_{y} \cdot \zeta \cdot z_{x}^{2}\right) \Omega_{z z}-2\left[2 \left(\zeta \cdot \zeta_{x} \cdot z_{x} \cdot z_{y}\right.\right. \\
& \left.+\zeta \cdot \zeta_{y} \cdot z_{x}^{2}+\zeta^{2} \cdot z_{x} \cdot z_{x y}+\left(2 \zeta \cdot \zeta_{x} \cdot z_{x} \cdot z_{y}+\zeta^{2} \cdot z_{y} \cdot z_{x x}\right)\right] \Omega_{z}^{2}-2\left[2 \left(\zeta \cdot \zeta_{x y} \cdot z_{x}\right.\right. \\
& \left.\left.+\zeta_{x}^{2} \cdot z_{y}+\zeta_{x} \cdot \zeta_{y} \cdot z_{x}+\zeta \cdot \zeta_{x} \cdot z_{x y}\right)+\left(2 \zeta_{x} \cdot \zeta_{y} \cdot z_{x}+\zeta \cdot \zeta_{y} \cdot z_{x x}+\zeta \cdot \zeta_{x x} \cdot z_{y}\right)\right] \Omega \cdot \Omega_{z} \\
& +\left[\left(\zeta_{x x x} \cdot z_{y}+3 \zeta_{x x y} \cdot z_{x}+3 \zeta_{x x} \cdot z_{x y}+3 \zeta_{x y} \cdot z_{x x}+3 \zeta_{x} \cdot z_{x x y}+\zeta_{y} \cdot z_{x x x}+\zeta \cdot z_{x x x y}\right)\right. \\
& -4\left(\eta_{x} \cdot \zeta_{x} \cdot z_{y}+\eta_{x} \cdot \zeta_{y} \cdot z_{x}+\eta_{x} \cdot \zeta \cdot z_{x y}+\eta_{x y} \cdot \zeta \cdot z_{x}\right)-2\left(2 \eta_{y} \cdot \zeta_{x} \cdot z_{x}+\eta_{y} \cdot \zeta \cdot z_{x x}\right. \\
& \left.\left.+\eta_{x x} \cdot \zeta \cdot z_{y}\right)+\left(\zeta_{x} \cdot z_{t}+\zeta_{t} \cdot z_{x}+\zeta \cdot z_{x x}\right)\right] \Omega_{z}-2\left(2 \zeta_{x} \cdot \zeta_{x y}+\zeta_{y} \cdot \zeta_{x x}\right) \Omega^{2}+\left[\zeta_{x x x y}\right. \\
& \left.+\zeta_{x t}-4\left(\eta_{x} \cdot \zeta_{x y}+\eta_{x y} \cdot \zeta_{x}\right)-2\left(\eta_{y} \cdot \zeta_{x x}+\eta_{x x} \cdot \zeta_{y}\right)\right] \Omega+\eta_{x x x y}+\eta_{x t}-4 \eta_{x} \cdot \eta_{x y} \\
& -2 \eta_{y} \cdot \eta_{x x}=0
\end{aligned}
$$

For simplifying the Equation 7 into a differential equation of $\Omega(z)$, the coefficient $\zeta \cdot z_{x}^{3} \cdot z_{y}$ of $\Omega_{z z z z}$ can be standard coefficient. After finishing this step, the other $\Omega(z)$ and it's coefficient of differential term all will have $\zeta \cdot z_{x}^{3} \cdot z_{y} \cdot T(z)$, and the $T(z)$ is $z$ 's undetermined function. During the operation arithmetic, it settled: Through differential and integral operation arithmetic, the $T(z)$ still record as $T(z)$; this way by using similar method for simplifying the question. The operation arithmetic can be used by three rules, which is for $\eta(x, y, t), \zeta(x, y, t), z(x, y, t)$ and $\Omega(z)$ :

(1) If $F(x, y, t)$ have the modality, $F(x, y, t)=F_{0}(x, y, t)+F(x, y, t) T(z)$, Then $T(x) \equiv 0$, based on the rule $T(z) \rightarrow$ $\Omega(z)$, that to replace $\Omega(z)$;

(2) If $F(x, y, t)$ have the modality: $F(x, y, t)=F_{0}(x, y, t) T(z)$, then $T(z) \equiv 1$; based on the rule $\frac{\Omega(z)}{T(z)} \rightarrow \Omega(z)$, that to replace $\operatorname{Omega}(z)$;

(3) If the equation $F(x, y, t) T(z)$ is determined by $T(z)=z_{0}(x, y, t)$, and $T(z)$ in it is reversible function, then $T(z)=z$; based on the rule $z \rightarrow T^{-1}(z)$ to replace. 
The coefficient of term $\Omega_{z} \cdot \Omega_{z z}$ :

$$
\zeta \cdot z_{x}^{3} \cdot z_{y} \cdot T(z)=-6 \zeta^{2} \cdot z_{x}^{2} \cdot z_{y} \Rightarrow \zeta=-\frac{1}{6} z_{x} \quad(T(z) \equiv 1)
$$

The coefficient of term $\Omega \cdot \Omega_{z z}$ :

$$
\zeta \cdot z_{x}^{3} \cdot z_{y} \cdot T(z)=-2\left(2 \zeta \cdot \zeta_{x} \cdot z_{x} \cdot z_{y}+\zeta \cdot \zeta_{y} \cdot z_{x}^{2}\right)
$$

Equation 8 substitute for Equation 9, then:

$$
z_{1}=\frac{x}{\varphi_{1}(y, t)}-\varphi_{2}(y, t) \quad\left(T(z) \equiv z_{1}\right)
$$

The same way can get the solution $z_{2}, z_{3}, z_{4}$ and $z_{5}$ of coefficient of term $\Omega_{z z z}, \Omega_{z}^{2}, \Omega \cdot \Omega_{z}$ and $\Omega^{2}$ :

$$
\begin{aligned}
& z_{2}=\frac{x}{\varphi_{1}(y, t)}-\varphi_{2}(y, t)\left(\operatorname{take} T(z) \equiv z_{2}\right) \\
& z_{3}=\frac{x}{\varphi_{1}(y, t)}-\varphi_{2}(y, t)\left(\operatorname{take} T(z) \equiv z_{3}\right)
\end{aligned}
$$

$\varphi_{1}=\varphi_{1}(y, t), \varphi_{2}=\varphi_{2}(y, t), \varphi_{3}=\varphi_{3}(y, t)$ is Any integral function about $y, t$ in the Equations $10,11,12$. It will be discussed the original similar form, similar argument and differential equation of Equation 7.

United the Equations 10, 11, 12:

$$
\zeta=-\frac{1}{6} z_{x}=-\frac{1}{6 \varphi_{1}(y, t)}, z=\frac{1}{6 \varphi_{1}(y, t)}-\varphi_{2}(y, t)
$$

Based on:

$$
\zeta=-16 z_{x} \Rightarrow\left\{\begin{array}{l}
\zeta_{y}=-\frac{\varphi_{1 y}(y, t)}{6 \varphi_{1}^{2}(y, t)}, \zeta_{t}=-\frac{\varphi_{1}(y, t)}{6 \varphi_{1}^{2}(y, t)} \\
\zeta_{x}=-\frac{1}{6} z_{x x}=0, \zeta_{x x}=-\frac{1}{6} z_{x x x}=0 \\
\zeta_{x y}=-\frac{1}{6} z_{x x y}=0, \zeta_{x x y}=-\frac{1}{6} z_{x x x y}=0 \\
\zeta_{x x x}=-\frac{1}{6} z_{x x x x}=0, \zeta_{x x x y}=-\frac{1}{6} z_{x x x y}=0
\end{array}\right.
$$

The coefficient of term $\Omega$ :

$$
\zeta \cdot z_{x}^{3} \cdot z_{y} \cdot T(z)=\zeta_{x x x y}+\zeta_{x t}-4\left(\eta_{x} \cdot \zeta_{x y}+\eta_{x y} \cdot \zeta_{x}\right)-2\left(\eta_{y} \cdot \zeta_{x x}+\eta_{x x} \cdot \zeta_{y}\right)
$$

Equations 13, 14 substitute for Equation 15:

$$
\eta=x \varphi_{4}(y, t)+\varphi_{5}(y, t)(T(z) \equiv 0)
$$

$\varphi_{4}=\varphi_{4}(y, t), \varphi_{5}=\varphi_{5}(y, t)$ is any integral function about $y, t$ in the Equation 16.

Equations 8, 9, 10, 11, 12, 13, 16 substitute for the Equation 7, and simplifying:

$$
\begin{aligned}
& \Omega_{z z z z}+\Omega_{z} \cdot \Omega z z+z\left[10 \Omega_{z z z}+\Omega \cdot \Omega_{z z}+\frac{7}{3} \Omega_{z}^{2}\right] \\
& +\frac{\varphi_{1}^{2}\left[x\left(\varphi_{1 t}-4 \varphi_{4} \varphi_{1 y}+2 \varphi_{1} \varphi_{4 y}\right)+\varphi_{21} \varphi_{1}^{2}-4 \varphi_{4} \varphi_{2 y} \varphi_{1}^{2} \varphi_{1}+2 \varphi_{1} \varphi_{5 y}\right]}{x \varphi_{1 y}+\varphi_{2 y} \varphi_{1}^{2}} \Omega_{z z} \\
& +\frac{2 \varphi_{1}^{2}\left(8 \varphi_{4} \varphi_{1 y}-\varphi_{1 t}+2 \varphi_{4 y} \varphi_{1}\right)}{x \varphi_{1 y}+\varphi_{2 y} \varphi_{!}^{2}} \Omega_{z}+\frac{6 \varphi_{1}^{6}\left(4 \varphi_{4 y} \varphi_{4}-\varphi_{4 t}\right)}{x \varphi_{1 y}+\varphi_{2 y} \varphi_{1}^{2}}=0
\end{aligned}
$$

The original similar form and similar argument of Equation 7 could be:

$$
\left\{\begin{array}{l}
U(x, y, t)=x \varphi_{4}(y, t)+\varphi_{5}(y, t)-\frac{1}{6 \varphi_{1}(y, t)} \Omega(z) \\
z=\frac{x}{\varphi_{1}(y, t)}-\varphi_{2}(y, t)
\end{array}\right.
$$

$\varphi_{1}=\varphi_{1}(y, t), \varphi_{2}=\varphi_{2}(y, t), \varphi_{4}=\varphi_{4}(y, t), \varphi_{5}=\varphi_{5}(y, t)$ is any integral function about in the Equation 18. Based on $\Omega(z)$ meet differential Equation 17, it is differential equation which has original similar form and similar argument, immediately it can change into the differential equation which has Paul Painlev's factors in some conditions. 


\section{The Similar Solution of $(2+1)$ D Breaking Soliton Equation}

In the Equation 17, take:

$$
\left\{\begin{array}{l}
\frac{\varphi_{1}^{2}\left[x\left(\varphi_{1 t}-4 \varphi_{4} \varphi_{1 y}+2 \varphi_{1} \varphi_{4 y}+\varphi_{21} \varphi_{1}^{2}-4 \varphi_{4} \varphi_{2 y} \varphi_{1}^{2} \varphi_{1}+2 \varphi_{1} \varphi_{5 y}\right]\right.}{x \varphi_{1 y}+\varphi_{2 y} \varphi_{1}^{2}}=C_{1}(z) \\
\frac{2 \varphi_{1}^{3}\left(8 \varphi_{4} \varphi_{1 y}-\varphi_{1 t}+2 \varphi_{4 y} \varphi_{1}\right)}{x \varphi_{1 y}+\varphi_{2 y} \varphi_{1}^{2}}=C_{2}(z) \\
\frac{6 \varphi_{1}^{6}\left(1 \varphi_{4 y}-\varphi_{4 t}\right)}{x \varphi_{1 y}+\varphi_{2 y} \varphi_{1}^{2}}=c_{3}(z)
\end{array}\right.
$$

Take $C_{1}(z)=\frac{z}{A}+B$, the coefficient of $x$ 's variable are equaled at the both side of equation, by considering the formula.

$$
\begin{gathered}
{\left[\varphi_{2 t} \varphi_{1}-4 \varphi_{4} \varphi_{2 y} \varphi_{1}^{2}\right] \varphi_{1}=\frac{z}{A} \varphi_{2 y}+B \varphi_{2 y}} \\
\varphi_{1}^{2}\left(\varphi_{1 t}-4 \varphi_{4} \varphi_{1 y}+2 \varphi_{1} \varphi_{4 y}\right)=\frac{z}{A} \varphi_{1 y}+B \varphi_{1 y}
\end{gathered}
$$

The analysis of formula 19:

$$
\left\{\begin{array} { l } 
{ \frac { 2 \varphi _ { 1 } ^ { 3 } ( 8 \varphi _ { 4 } \varphi _ { 1 y } - \varphi _ { 1 t } + 2 \varphi _ { 4 y } \varphi _ { 1 } ) } { x \varphi _ { 1 y } + \varphi _ { 2 y } \varphi _ { 1 } ^ { 2 } } = C _ { 2 } ( z ) \equiv 0 } \\
{ \frac { 6 \varphi _ { 1 } ^ { 6 } ( 4 \varphi _ { 4 y } \varphi _ { 4 } - \varphi _ { 4 } ) } { x \varphi _ { 1 y } + \varphi _ { 2 y } \varphi _ { 1 } ^ { 2 } } = C _ { 3 } ( z ) \equiv 0 }
\end{array} \Rightarrow \left\{\begin{array}{l}
\varphi_{1}=y^{\frac{1}{4}}+t^{\frac{1}{2}} \\
\varphi_{4}=-\frac{y}{4 t}
\end{array}\right.\right.
$$

Equation 22 substitute for equation:

$$
\text { (21) } \frac{z}{A}+B=2\left(y^{\frac{1}{4}}+t^{\frac{1}{2}}\right)^{2}\left(y^{\frac{3}{4}} t^{-\frac{1}{2}}-y^{1} t^{-1}-y^{\frac{3}{4}} t^{-\frac{3}{4}}\right)
$$

Commend $A=a_{0}, B=0$, united and operated Equations 13, 23:

$$
\left\{\begin{array}{l}
z=2 y^{\frac{3}{4}} t^{-1} a_{0}\left(y^{\frac{1}{4}}+t^{\frac{1}{2}}\right)^{2}\left(t^{\frac{1}{2}}-y^{\frac{1}{4}}-t^{\frac{1}{4}}\right) \\
\varphi_{2}=x\left(y^{\frac{1}{4}}+t^{\frac{1}{2}}\right)^{-1}-2 a_{0}\left(y^{\frac{1}{4}}+t^{\frac{1}{2}}\right)^{2}\left(y^{\frac{3}{4}} t^{-\frac{1}{2}}-y^{1} t^{-1}-y^{\frac{3}{4}} t^{-\frac{3}{4}}\right)
\end{array}\right.
$$

Equations 23, 24 substitute for Equation 20:

$$
\begin{aligned}
& \varphi_{5}(y, t)=\frac{3}{8} a_{0} y^{3} t^{-2}+\frac{3}{22} a_{0} y^{\frac{11}{4}} t^{-\frac{7}{4}}+\frac{1}{11} a_{0} y^{\frac{11}{4}} t^{-\frac{5}{4}}-\frac{8}{11} a_{0} y^{\frac{11}{4}} t^{-2}+\frac{7}{22} a_{0} y^{\frac{11}{4}} t^{-\frac{3}{2}}-\frac{1}{5} a_{0} y^{\frac{5}{2}} t^{-\frac{3}{2}} \\
& \left.-\frac{9}{20} a_{0} y^{\frac{5}{2}} t^{-\frac{5}{4}}+\frac{1}{1}\right)(5) y^{\frac{2}{2}} t^{-1}\left(\frac{3}{2} a_{0}-1\right)+\frac{1}{10} a_{0} y^{\frac{5}{2}} t^{-\frac{3}{4}}+\frac{8}{9} a_{0} y^{\frac{9}{4}} t^{-\frac{5}{4}}-y^{\frac{9}{4}} t^{\frac{3}{4}}\left(\frac{2}{3}+a_{0}\right)+\frac{2}{9} a_{0} y^{\frac{9}{4}} t^{-1} \\
& +\frac{1}{3} y^{\frac{9}{4}} t^{-\frac{1}{2}}\left(\frac{2}{3}-\frac{1}{2} a_{0}\right)+\frac{1}{8} a_{0} y^{2} t^{-\frac{7}{4}}+\frac{5}{4} a_{0} y^{2} t^{-\frac{3}{4}}-\frac{3}{2} a_{0} y^{2} t^{-\frac{1}{2}}-\frac{1}{4} a_{0} y^{2}-\frac{3}{3} a_{0} y^{\frac{7}{4}} t^{\frac{1}{2}}+\frac{3}{14} a_{0} y^{\frac{7}{4}} t^{\frac{1}{4}} \\
& +\frac{2}{7} a_{0} y^{\frac{7}{4}} t^{-\frac{1}{2}}-a_{0} \frac{4}{7} y^{\frac{7}{4}}-\frac{2}{5} y^{\frac{5}{4}} t^{-\frac{1}{2}}-\frac{1}{2} y t^{-\frac{1}{4}}+\frac{1}{2} y-\frac{1}{3} y^{\frac{3}{4}} x t^{-1}-x y^{-\frac{1}{2}} t^{-\frac{1}{2}}
\end{aligned}
$$

The similar solution of $(2+1) \mathrm{D}$ breaking soliton equation:

$$
\left\{\begin{array}{l}
U=x-\frac{y}{4 t}+\varphi_{5}(y, t)-\frac{1}{6\left(y^{\frac{1}{4}}+t^{\frac{1}{2}}\right)} \Omega(z) \\
z=2 a_{0}\left(y^{\frac{1}{4}}+t^{\frac{1}{2}}\right)^{2}\left(y^{\frac{3}{4}} t^{-\frac{1}{2}}-y^{1} t^{-1}-y^{\frac{3}{4}} t^{-\frac{3}{4}}\right)
\end{array}\right.
$$

$\Omega(z)$ meets the differential equation which have the fourth type of Paul Painlev's Factor, and $\varphi_{5}(y, t)$ based on the Equation 25, in the Equation 26.

$$
\Omega_{z z z z}+\Omega_{z} \cdot \Omega_{z z}+z\left[10 \Omega_{z z z}+\Omega \cdot \Omega_{z z}+\frac{7}{3} \Omega_{z}^{2}\right]+\frac{z}{A} \cdot \Omega_{z z}=0
$$

\section{Conclusion}

Because of $\varphi_{i=1-5}(y, t)$ is Any integral function about $y, t$, the original similar form and the similar argument differential equation of $(2+1)$ - dimensional breaking soliton equation have different modality in the different conditions of problems. For reducing difficulty and measure of operation arithmetic, take some special assignment for $A, B$, and different assignment will caused Equation 26 have different modalities during the derivation process of similar solute the Equation 26. It is said that all the rules and the way of taking assignment which is used in derivation process could not affect the way of thinking and the method of operation arithmetic of similar solution, and also could not affect the differential equation about the original similar form and similar argument. The contributing to offered a other way of thinking and operation arithmetic method for equation integrability analysis, The cloning transform and Soliton solution of $(2+1)$ - dimensional nonlinear partial differential equations. 


\section{Reference}

Chun-Long, Zheng, \& Jie-Fang, Zhang. (2002). Similarity reductions and analytic solution for the (2+1) dimensional Camassa-Holm equations. Acta Phys. Sin., 51(11), 2430. Reprinted from http://159.226.36.45/Jwk_aps/CN/Y2002/V51/I11/2430

Gu, C. H. (1990). Soliton Theory and its Application (p. 160). Hangzhou: Zhejiang Publishing House of Science and Technology.

Guo, B. L. (1998). Nonlinear Evolution Equations (p. 146, pp. 169-187). Shanghai: Shanghai Scientific and Technological Education Publishing House.

Hang-Yu, Ruan, \& Yi-Xin, Chen. (2003). Study on soliton interaction in the (2+1) - dimensional NizhnikNovikov-Veselov equation. Acta Phys. Sin., 52(6), 1318. Reprinted from http://159.226.36.45/Jwk_aps/CN/Y2003/V52/I6/1318

Jie-Fang, Zhang, \& Guan-Ping, Guo. (2003). Novel multi-soliton solutions of the breaking soliton equation. Acta Phys. Sin., 52(10), 2362. http://159.226.36.45/Jwk_aps/CN/Y2003/V52/I10/2362

Jie-Fang, Zhang, Wen-Hua, Huang, \& Chun-Long, Zheng. (2002). Coherent soliton structures of a new (2+1) dimensional evolution equation. Acta Phys. Sin., 51(12), 2682. Reprinted from http://159.226.36.45/Jwk_aps/CN/Y2002/V51/I12/2682

Jun, Lei, Song-Hua, Ma, \& Jian-Ping, Fang. (2011). Multiple quadrate soliton solutions and chaotic behaviours of (2+1)-dimensional breaking soliton equation. Acta Phys. Sin., 60(5), 050302. Reprinted from http://159.226.36.45/Jwk_aps/CN/Y2011/V60/I5/050302

Li, Y. S. (1999). Soliton and Integral System (pp. 25 - 31). Shanghai: Shanghai Scientific and Technological Education Publishing House.

Xiao-Xia, Cao, Song-Hua, Ma, Qing-Bao, Ren et al. (2012). Multiple solitoff solutions and the evolution of (2+1)dimensional breaking soliton equation. Acta Phys. Sin., 61(14), 0140505. Reprinted from http://159.226.36.45/Jwk_aps/CN/Y2011/V60/I5/050302 